\section{Les meilleurres Rivières du Bassin de la Loire}

L'ouvrage, récemment paru. de M. J.-A. Montpellier, Les Distributions publiques l'énergie électriques, montre quil y avait en France, au 1er janvier 1907, 1413 usines génératrices d'électricité, alimentant 2912 localités, en présence de 824 usines à gaz alimentant 1209 localités or, d'une part, si lon tient compte des époques auxquelles remonte l'usage pratique de l'industrie électrique et de l'industrie du gaz, on est encore plus frappé du développement rapide de la première. D'autre part, l'auteur, dans un classement rigoureux, démontre que, sur les 1413 usines électriques, 831 sont exclusivement hydrauliques (donc près des deux tiers et encore To plus que d'usines à gaz) et que 169 utilisent simullanément une chute d'eau et différents genres de moteurs do secours (vapeur, gaz pauvre, pétrole); finalement, 413 usines électriques seulement nutilisent aucun moteur hydraulique

M H. Bresson estime que, après cet exposé, il doit êtro intéressant de connaitre les rivières de France qui méritent deetre ainsi utilisées, surtout quand on songe que nous sommes tributaires de l'étranger pour 25 pour 100 environ de notre consommation en houille et pour la totalité du pétrole; aussi, dans La Revue Electrique du 15 février dernier, a-t-il dressé un lexique des meilleures rivières du bassin de la Loire.

Pour ce lexique, $M$. Bresson a adopté l'ordre alphabétique, mais, en terminant, il produit une Table méthodique dans laquelle les rivières sont rangées selon leur ordre d'affluents par rapport à la Loire. Ce lexique donne la longueur totale du cours d'eau, les altitudes de sa source et de son embouchure. Dans chaque département traversé, d'amont vers l'aval, on y trouvéra les localités riveraines de plus de 1000 habitants avec leurs populations, toujours selon les documents administratifs. Chaque article de ce lexique est lerminé par le renseignement essentiel suivant : le nombre des usines ayant existé à la date donnée, et la plus puissante d'entre elles avec la hauteur de sa chute d'eau Il est encore entendu que cette évaluation est celle des anciennes roues de moulin, seules employées généralement à ces dates parfois assez reculées.

Les statistiques administratives à consulter sont établies par département, mais il est plus logique d'adopter pour cadre le bassin fluvial; il y en a quatre rrincipaux en France, plus les bassins côtiers, ou partagés avec l'étranger, qui formeraient une cinquieme partie.

Le présent bassin, celui de la Loire, est le plus central de la France. Dans une statistique, on ne peut s'appuyer que sur ce qui a existé; en conséquence, le chuix des rivières a été fait ici d'après la puissance d'un moteur hydraulique ayant produit, à une époque qui sera toujours donnée, une force minima de 25 chevaux. Celle-ci est souvent suffisante pour une entreprise d'une distribution d'énergie électrique dans un chef-lieu de canton d'une population fréquente de 1500 habitants. M. Bresson en a relevé de nombreux exemples dans une etude poursuivie depuis quatre annces dans la région normande $\left(^{\star}\right)$.

Certains petits cours d'eau, déjà utilisés pour la production de l'énergie électrique, ne figurent pas dans ce lexique, car l'auteur n'a pas la preuve que le criterium de 25 chevaux soit atteint.

M. J. BLondin, rédacteur en chef de la Revue Electrique, a fait précéder le lexique de M. Bresson des judicieuses considérations suivantes:

() La Houille verte: Hise en valeur des moyennes el basses Blanch d'eau en France liunod el Pinat, Paris. - Voir aussi La Houille Blanche de mars 1904
“Les puissances des chutes d'eau que signale M. H. Bresson dans son lexique des meilleures rivières du bassin de la Loire n'ont, certes, pas l'importance des chutes des Alpes. Ces petites chutes n'en ont pas moins un intérêt général pour le déveloprement des applications de l'élec-

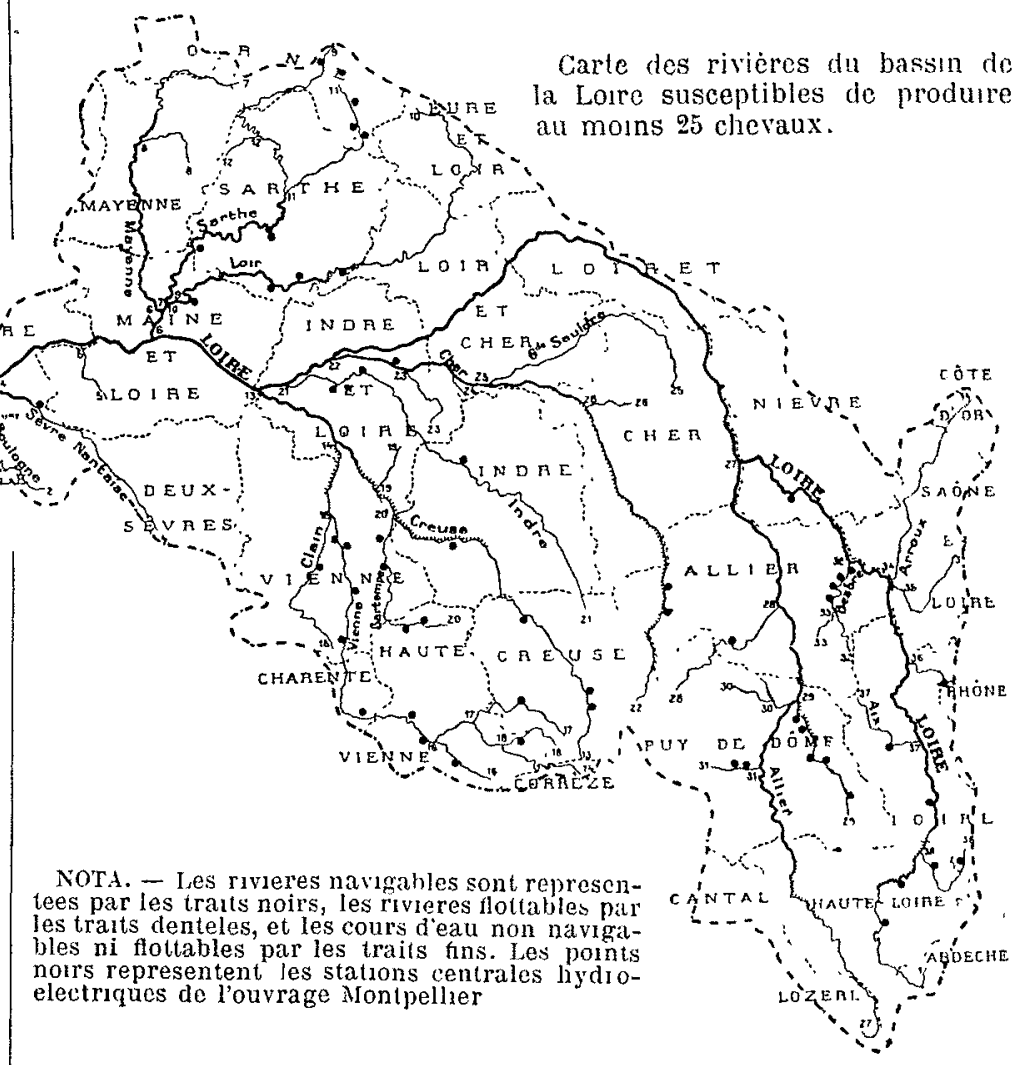

tricité. Elles peuvent, en eflet, comme lo montront de nombreux exemples signalés par l'auteur dans un ouvara antéricur, être économiquement utilisées pour la distribution par l'électricité de l'éclairage et de la force motrico dans les villages. Elles permettent, dès lors, de familiariser nos populations agricoles avec les applications do l'olestricité. Et, si l'on songe que, d'une part, l'agriculture est la principale ressource de la France, que, d'autre part, les grandes Compagnies de distribution espèrent trouver le placement d'une bonne partie de la force motrice dont elles disposent dans des opérations agricoles, telles que l'irrigation, le labourage, etc., on peut alfirmer que toute installation électrique faite dans les campagnes constitue, si fauble que soit sa puissance, un premier jalon dans la voie du développement de la grande industrie ólectrique. En rassemblant et condensant los nombreuses indications éparses dans les documents officiels et permettant ansi leur utilisation par les industriels, M. Bresson fail donc ceuvre utile n.

\section{THÉORIE DU DÉVERSOIR}

Notes de M. Boussinesq, de l'Académie des Sciences (Suile)

\section{Théorie approchée de l'écoulement sur un déversoir vertical en mince paroi, sans contraction latérale et à nappe libre (").}

I. - J'ai donné, dès 1887 , une théorie approchée de l'écoulement de l'eau sur un déversoir vertical ou incliné, à mince paroi et sans contraction latérale, mais en supposant demandée directement à l'observation la mesure de la con- 
traction inférieure $c$ de la nappe déversante, rapport, à la hauteur $h$ de charge, du relèvement total $\varepsilon$ qu'éprouve la face inférieure de la nappe après s'êtredétachée du seuil horizontal. Ce relèvement $\varepsilon$ est dû à la convergence mutuelle des filets fluides, dont les plus hauts, dirigés vers l'aval, sont encore presque horizontaux sur le seuil, tandis que les plus bas, ascendants contre la paroi antérieure du barrage, sont, d'ordinaire, en quittant le seuil, verticaux comme elle, et peuvent même, si la paroi est munie d'une large armature horizontale braquée vers l'amont, arriver au seuil avec une vitesse horizontale inverse de celle des filets supérieurs, avec lesquels ils font alors un angle de près de deux droits.

Or comme, dans cette question, inabordable jusqu'içi rationnellement, une solution très approchée paraît résulter de l'hypothèse simple de l'existence, dans la nappe, d'une section contractée presque verticale, à la traversée de laquelle tous les filets fluides contenus dans un même plan longitudinal seraient parallèles avec centre commun de courbure, il est naturel de compléter cette hypothèse, par une autre non moins simple, en admettant, du moins pour les déversoirs très hauts où le mouvement est insensible au fond et où le phénomène acquiert son minimum de complication, que la contraction inférieure $c$ est, pour chaque valeur $K$ du rapport de la hauteur $h$ ' de l'eau sur la section contractée à la hauteur $h$ de charge, sensiblement proportionnelle à la convergence des filets sur le seuil et, par conséquent, dans le cas du déversoir vertical, moitié de la valeur maximum $C$ qu'elle atteint dans le cas du déversoir avec armature.

II. - J'ai obtenu, dans les articles cités, comme expression du débit $q$ de l'unité de longueur du déversoir, la formule $q=m h \sqrt{2 g h}$, où le coefficient de débit $m$ est, pour chaque valeur du rapport $K$ :

avec $k=\sqrt{\frac{1+X}{1-c}} \quad$ et $\left.\quad \mathrm{f}(k)=\left(k+k^{2}\right) \log \frac{1}{k},\right\}$

le paramètre auxiliaire $k$ se trouvant ainsi relié à la contraction inférieure $c$ et à la dénivellation relative :

$$
\mathrm{I}-K=\frac{h-h^{\prime}}{h}
$$

sur la section contractée. D'ailleurs, si l'on appelle $\%$ la valeur de $k$, pour mème dénivellation relative $\mathrm{I}-K$, sur le déversoir avec armature où la contraction inférieure est $C$, il existe entre $C$ et $x$ la formule assez simple, fournie par le principe des quantités de mouvement :

$$
(1-C)^{2}(1+x)^{3}(1-x)=\mathrm{I}, \text { avec } x=\sqrt{\frac{1-K}{1-C}}
$$

et la différentiation de celles-ci par rapport à $K$ donne assez aisément, pour la dérivée $C^{\prime}$ de $C$, après élimination de la dérivée, $x^{\prime}$, de $x$ :

$$
C^{\prime}=\frac{2 x-1}{x(2-x)} \text {. }
$$

Or, pour le déversoir vertical, la contraction inférieure $c$ est supposée la moitié de $C$ et, par suite, sa dérivée $c$, la moitié de $C^{\prime}$. L'on aura done, d'après (2) et (3):

$$
c=\frac{1}{2}-\frac{1}{2 \sqrt{(1+x)^{3}(1-x)}}, c^{\prime}=\frac{2 x-1}{2 x(2-x)} .
$$

Enfin, la seconde équation (1), écrite $(1-c) k^{2}=1-K$ et différentiée en $K$, donnera elle-même :

$$
2(\mathrm{I}-c) k k^{\prime}=-\left(\mathrm{I}-k^{2} e^{\prime}\right) .
$$

III. - On sait que l'écoulement sur le déversoir devient constant, par un abaissement suffisant de l'eau en aval, qui fait décroitre $K$ (supposé d'abord égal à I), à l'instant où, $K$ diminuant ainsi peu à peu (et $k$ augmentant), l'expres. sion (I) du coefficient $m$ de débit devient maximum. Il y a donc lieu d'égaler à zéro la dérivée de $m$; ce qui, vu (5), donne pour déterminer $k$, l'équation:

$$
\mathbf{f}^{\prime}(k)=-\left[3 \mathrm{f}(k)-k \mathrm{f}^{\prime}(k)\right] k c^{\prime} .
$$

Cette équation se résout par approximations successives en remarquant: d'une part, que le premier membre s'annule pour la valeur $k_{0}=0,46854$ de $k$ rendant $f(k)$ maximum et, le second, pour celle, $k_{1}$, qui annule $c$ ' ou corres. pond $\dot{a} x=\frac{1}{2}$ et au maximum $\frac{1}{2}-\frac{2}{\sqrt{27}}=0,1151$ de $c$; d'autre part, que cette seconde valeur $k$ est presque identique à la première, à cause de l'égali té des deux expressions $(\mathrm{I}-C) \mathrm{x}^{2},(\mathrm{I}-c) k^{2}$ de $\mathrm{I}-K$, d'oú il résulte :

$$
k=\% \sqrt{\frac{1-2 c}{I-c}} \text { et } k_{1}=\frac{1}{2} \sqrt{\frac{0,7698}{0,8849}}=0,46634 \text {. }
$$

J'ai obtenu ainsi, à une deuxième approximation : $k=0,4694, \quad x=0,5033, \quad c=0$, I I 5 I, et $m=0,4342$

IV. - Mais on peut encore, à la condition de choisir\% comme variable auxiliaire, essayer successivement diverses valeurs de $x$, par exemple $\%=0,5032, \quad 0,5033,0,5034$. Pour chacune d'elles, les formules (4) font connaitre $c$ et $c^{\prime}$, puis la première formule (7) donne $k$ et rend, par conséquent, évaluables les deux fonctions :

$$
\mathrm{f}(k)=\left(k+k^{2}\right) \log \frac{\mathrm{I}}{k}, \mathrm{f}^{\prime}(k)=(1+2 k) \log \frac{\mathrm{I}}{k}-(\mathrm{I}+k)
$$

On peut donc enfin avoir les deux membres de (6) et l'excédent du premier sur le second. Or, les trois essais $x=0,5032,0,5033,0,5034$ donnent respectivement, pour cet excédent, o,0003o, 0,00006, - o,ooorg. Donc, la racine cherchée se trouve comprise entre o,5033 et 0,5034 ; et un calcul par parties proportionnelles donne enfin sa vraie valeur, o, 503324 , qui se vérifie ensuite directement. L'on trouve alors :

$$
k=0,46945, c=0,115 \mathrm{r}, \quad m=0,43422 ;
$$

ce qui confirme pleinement les calculs de deuxième approximation.

Les expériences de $M$. Bazin ont donné environ $c=0,112$ et $m=0,43$. 
Théorie approchée de l'écoulement sur un déversoir vertical en mince paroi, sans contraction latérale, et à nappe noyée en-dessous $\left(^{\star}\right)$.

I. - Les mêmes principes qui m'ont permis, dans une Note du 24 mars 1907 , de donner une théorie approchée de l'écoulement sur un déversoir à nappe libre inférieurement, s'appliquent lorsque la nappe est noyée en dessous, pourvu qu'on assimile à du fluide mort, ayant sa pression hydrostatiquement croissante de haut en bas et donnée directement au niveau du seuil, la masse liquide qui tourbillonne sous la nappe. La hauteur de charge étant appelée $h$, et le poids de l'unité de volume liquide $\rho g$, nous désignerons par $N^{\prime}$ pgh cette pression au niveau du seuil, supposée ici constante comme $h$; de sorte que $N^{\prime}$ sera un nombre donné $\left({ }^{\star}\right)$.

Les raisonnements qui m'avaient conduit aux équations (1) et (2) de la Note citée donnent ici respectivement :

$$
m=(r-c)^{\frac{3}{2}}\left(n k-n^{3} k^{3}\right) \frac{\log k}{k-\mathrm{I}}
$$

dyec:

$$
n=\sqrt{\frac{\mathrm{I}-N^{\prime}}{\mathrm{I}-c}} \text { et } k=\sqrt{\frac{\mathrm{I}-K}{\mathrm{I}-\bar{N}}} ;
$$

$$
\begin{aligned}
& \frac{\mathrm{I}}{\mathrm{I}-2 c}=\mathrm{I}+k-\nu^{2} k^{3}\left(\mathrm{I}+\frac{k}{2}\right) \\
& \nu=\sqrt{\frac{\mathrm{I}-N}{\mathrm{I}-2 c}}=n \sqrt{\frac{\mathrm{I}-c}{\mathrm{I}-2 c}} .
\end{aligned}
$$

D'ailleurs, la différentiation en $K$ de l'équation (2) donne pour la dérivée $c$ de $c$, après élimitation des dérivées de $k$ et de $\vee$ :

$$
c^{\prime}=\frac{v^{2} k^{2}(3+2 k)-1}{4^{2} k(1+k)} .
$$

II. - L'écoulement constant cherché, produit par un abaissement suffisant du niveau d'aval (avec $N^{\prime}$ censé invariable), s'obtient encore en rendant maximum l'expression (1) du coefficient $m$ de débit, lorsque $K$ y décroît à partir de r. Il y a donc lieu d'annuler la dérivée de $m$ en $K$ (ce qui conduit à faire varier $k ; c, n$ ); et il vient ainsi, au lieu de l'équation (6) de la Note citée :

$$
1-\frac{1}{2}\left(\frac{\mathrm{I}}{n^{2} k^{2}}-\mathrm{I}\right)\left(\frac{\mathrm{I}}{\log k}+\frac{\mathrm{I}}{1-k}\right)=c^{\prime} .
$$

Pour les pressions relatives sous la nappe, $N^{\prime}$, les plus usuelles, cette équation se résout encore par approximations successives, en continuant à remarquer, d'une part, que le premier membre s'annule pour la valeur $k_{0}$ de $k$ rendant $m$ maximum dans l'hypothèse de $c$ et $n$ constants et, le second membre, pour celle, $k_{1}$, correspondant au maximum de $c$; d'autre part, que cette seconde valeur $k_{1}$ est voisine de la première $k_{0}$ : en sorte que, dans tout l'intervalle séparant $k_{0}$ de $k_{1}$ et auprès, $K, k$ varient assez peu pour laisser négligeables les petites variations (du second ordre) de $c$, de $n$ et de v aux environs de leurs maxima respectifs $c_{1}, n_{1}, v_{1}$.

(") Séance du 1 er juillet 1907

(1) La notation plus simple $\mathrm{Negh}$ est reservée pour désigner la p'est-à-direore sous la nappe, mais au sommet des filets inférieurs, $N^{\prime}-N+$ à-dire à la hauteur $\varepsilon$ ou $c h$ au-dessus du seuil. On a donc $N^{\prime}=N+c$
Par exemple, l'annulation du second membre de (3), et puis du premier de (4), en passant par les deux relations (2), donne successivement :

$\begin{array}{rlllllll}\text { pour } \quad k_{1}= & 0,3 & 0,4 & 0.5 & 0,6 & 0,7 & 0,8 & 0,9 \\ \boldsymbol{y}^{2} & =3,086 & \mathbf{1}, 645 & \mathrm{1} & 0,661 & 0,464 & 0,340 & 0,257 \\ c & =0,085 & 0,107 & 0,128 & 0,140 & 0,163 & 0,179 & 0,193 \\ n^{2}= & 2,800 & 1,447 & 0,853 & 0,548 & 0,373 & 0,2660, & 196 \\ k_{0} & =0,288 & 0,304 & 0,507 & 0,627 & 0,751 & 0,879 & 1,008 \\ k_{0}-k_{1}=-0,012-0,006 & 0,007 & 0,027 & 0,051 & 0,079 & 0,108 \\ N^{\prime} & =-1,563-0,291 & 0,256 & 0,532 & 0,688 & 0,782 & 0,842\end{array}$

III. - On voit que, dans ce très large intervalle où la pression $N^{\prime} \mathrm{pgh}$, sous la nappe, varie en tout, de part et d'autre de zéro, de près de deux fois et demie la pression hydrostatique $\rho g h$ et finit par dépasser ses 84 centièmes, c'est-à-dire par approcher presque du maximum $p g h$ pour lequel cesserait tout écoulement, l'écart $k_{0}-k_{1}$ des deux valeurs de $k$ annulant respectivement le premier et le second nombre de (4) reste presque toujours une fraction peu sensible de $k_{0}$, pour n'atteindre qu'à la fin le dixième environ de $k_{0}$.

La racine $k$ de (4) se trouvant ainsi voisine de $k_{1}$ et de $k_{0}$, les deux nombres de (4) deviennent très sensiblement proportionnels: le premier, à $k-k_{0}$, le second, à $k-k_{1}$. L'équation (4) est, dès lors, du premier degré; et sa résolution donne pour $k$ l'expression :

$$
k-k_{0}+\frac{\frac{3}{2} A k_{0}}{1-\frac{3}{2} A k_{0}}\left(k_{0}-k_{1}\right),
$$

où $A$ est une fonction de $k_{0}$ lentement croissante, qui prend les valeurs :

$A=0,367 \quad 0,368$ o,370 0,37 г $0,3720,373 \quad 0,374 \quad 0,375$ pour :

$\begin{array}{llllllll}k_{0}=0,3 & 0,4 & 0,5 & 0,6 & 0,7 & 0,8 & 0,9 & \text { I }\end{array}$

IV. - Après avoir obtenu au préalable, par l'annulation du premier nombre de (4) pour un certain nombre de valeurs de $k_{0}$, les valeurs de $n^{2}$ correspondantes, et par l'annulation de $c$ pour un certain nombre de valeurs de $k_{1}$, celles de $v^{2}, c$ et $n^{2}$, l'égalisation des $n^{2}$, de part et d'autre, m'a donné à très peu près les valeurs simultanées (c'est-àdire correspondant à une même pression inférieure $N^{\prime} \mathrm{pgh}$ ) de $k_{0}, k_{1}, c, n$ et, par suite, de $k$. L'expression (I) du coefficient $m$ du débit devenait, dès lors, calculable; et, en la divisant finalement par le coefficient théorique de débit $m^{\prime}=0,4342$ du déversoir à nappe libre, i'ai pu former le tableau suivant, où les valeurs successives de $k_{0}$ sont 0,325 , $0,35,0,40,0,45,0,50,0,60,0,70,0,80,0,90$ :

\section{Pour :}

$N^{\prime}=-0,961-0,668-0,24^{5} \quad 0,0390,2390,492 \quad 0,6390,7330,79^{5}$

$m=\begin{array}{lllllllllll}0,530 & 0,509 & 0,472 & 0,440 & 0,413 & 0,369 & 0,333 & 0,305 & 0,280\end{array}$

$\frac{m}{m^{\prime}}=\quad 1,221 \quad 1,173 \quad 1,088 \quad 1,0140,951 \quad 0,849 \quad 0,768 \quad 0,701 \quad 0,646$

Or, M. Bazin a trouvé expérimentalement, comme expressions du même rapport $\frac{m}{m}$ de coefficients de débit, les formules empiriques: 


$$
\frac{m}{m^{\prime}}=1,0 \mathrm{I}-0,245 N^{\prime}\left(\mathrm{I}+\frac{N^{\prime}}{5}\right)
$$

pour $N^{\prime}$ compris environ entre $-0,9$ et 0,3 ;

$$
\frac{m}{m^{\prime}}=1-0,235 N^{\prime}\left(1+N^{\prime}\right)
$$

pour $N^{\prime}$ compris entre o,3 et 0,6 ;

$$
\frac{m}{m^{\prime}}=\text { (environ) } \mathrm{I}, 05 \sqrt[3]{\mathrm{I}-N^{\prime}}
$$

pour $N^{\prime}$ supérieur à 0,6 ,

dont la première concerne les nappes noyées seulement en dessous, ou dégagées du remous d'aval, tandis que les deux dernières s'appliquent aux pressions $N^{\prime} \rho g h$ ne se produisant que sous lès nappes' atteintes par le remous d'aval.

Pour les valeurs ci-lessus de $N^{\prime}$, ces formules donnent:

$$
\frac{m}{m}=\left\{\begin{array}{lllll}
1,200 & 1,152 & 1,067 & \text { 1,000 } & 0,930 \\
0,827 & 0,748 & 0,676 & 0,619 &
\end{array}\right.
$$

$V$. - Les valeurs théoriques sont, comme on voit, plus fortes. On se l'explique en observant que les pertes de force vive translatoire négligées par notre théorie, et employées à faire tourbillonner sous la nappe une masse fluide que nous supposons ici en repos, doivent être beaucoup plus grandes quand cctte masse est de l'eau que lorsqu'elle est de l'air et, par conséquent, en réduisant tout à la fois la vitesse moyenne et le débit, atténuer bien plus les numérateurs $m$ que le dénominateur $m$ '.

Toutefois, si l'on songe que nos calculs n'ont emprunté à l'expérience aucune donnée quantitative, aucun élément numérique, on sera peut-être surpris de voir l'excédent de leurs résultats sur ceux de l'observation rester parfois audessous de 0,02 et n'approcher de 0,03 que pour les valeurs de $N$ ' supérieures à 0,7 , pour lesquelles le débit constant ou maximum ne se réalisait peut-être pas tout à fait, la dénivellation entre l'amont et l'aval y étant très faible.

VI. - Au lieu d'évaluer la racine $k$ par la méthode précédente d'approximation, qui fait intervenir les quantités auxiliaires $k_{0}, k_{1}$, et qui, d'ailleurs, tomberait en défaut pour les valeurs de $N$ 'voisines de l'unité, on yeut aussi, mais au prix d'une bien plus grande longueur des calculs, exprimer en fonction de $k$ et de $N^{\prime}$ tous les autres paramètres, notamment $c$ et $n$, puis, comme $N^{\prime}$ est connu, essayer successivement différentes valeurs de $k$.

A cet effet, l'élimination de v entre les équations (2), puis la seconde ( 1 ), donnent aisément l'équation 6 suivante:

$$
c=\frac{k}{2(\mathrm{I}+k)}\left[\mathrm{I}-\left(\mathrm{i}-N^{\prime}\right) k^{2}\left(\mathrm{I}+\frac{k}{2}\right)\right], n=\sqrt{\frac{\mathrm{I}-N^{\prime}}{\mathrm{I}-c}}
$$

On peut donc, pour des valeurs arbitraires de $k$, connaître exactement $c, v, c^{\prime}, n$ et évaluer la différence entre les deux membres de, l'équation (4). Quelques tâtonnements, suivis de l'ordinaire interpolation par parties proportionnelles, conduiront"donc à la vraie racine $k$ de cette équation.

Mais on peut observer aussi que l'équation (4), quand on y remplace $c^{\prime}$ par sa valeur (3), est linéaire par rapport aux inverses de $\hat{n}^{2}, v^{2}$, et qu'elle le devient par rapporı à celui de I $-N$, après substitution de la valeur (6) de $c$ dans les expressions de ces inverses. Donc l'équation (4) sera linéaire en I $-N^{\prime}$ ou en $N^{\prime}$, et résoluble sans radicaux, si l'on se donne $k$ au lieu de $N^{\prime}$; après quoi $m$ se déduira de la première (1).

Par exemple, l'hypothèse $k=$ I conduit à $N^{\prime}=\frac{4}{5}=0,8$, $m=\frac{V \overline{5}}{8}=0,2795$, tandis que, pour $N^{\prime}=0,8$, une légère $^{\prime}$ extrapolation de la table ci-dessus de valeurs approchées de $m$ donne $m=0,278$, ou $\frac{\mathrm{I}}{186}$ sculement en moins.

La méthode approchée suffit donc parfaitement jusqu'àla limite $k=\mathrm{t}$, ou $N^{\prime}=0,8$, au delà de laquelle les filets fluides n'ont plus quine courbure insensible sur la section contractée et, laissant la pression y varier hydrostatiquement, permettent, sans calcul de maximum, une évaluation directe beaucoup plus simple de $m$ en fonction de $N^{\prime}\left({ }^{*}\right)$.

\section{LE MOIS HYDRO-ÉLECTRIQUE \\ ACADÉMIE DES SCIENCES}

\section{MÉCANIQUE ET ÉLEGTRIGITÉ}

Sur un hygroscope électrique de grande sensibilité. Note de M. J. Pionchon, séance du i 3 avril 1908 .

Dans un circuit, comprenant un galvanomètre sensible à miroir et une pile ou une dynamo d'une force électromotrice d'une centaine de volts, un tube de verre, du calıbre d'un tube à essais et d'une dizaine de centimètres de longueur, se trouve inséré par l'intermediaire de couches d'argenture recouvrant sa face interne et sa face externe, depuis l'extrème bord à un de ses bouts jusqu'à $\mathrm{I} \mathrm{cm}$. environ de distance de l'autre bout. Tel est l'appareil auquel l'auteur a reconnu qu'on pouvalt faire jouer le rôle d'un hygroscope d'une très grande déltcatesse.

La partie sensible est la section terminale du tube à l'extrémité oú les couches d'argenture ne sont séparées que par l'épaisseur du verre. Il suftit d'approcher à quelques millimètres de cette section un corps quelconque émettant des traces de vapeur d'eau pour roir le spot du galvanomètre, tout d'abord parfattement immobile, se déplacer vivement sur la règle, annonçant ansi que la surface de la tranche terminale du tube est devenue conductrice. Cet effet cesse d'ailleurs aussitôt qu'on éloigne le corps qui en a été la cause et le spot reprend sa position d'équilibre primitive. La rapidité avec laquelle la modification de la surface du verre se produit on se dissipe permet de penser qu'elle est le fait de traces de vapeur tout à fait impondérables.

Il est préférable que la section terminale du tube, au lieu d'être bordée a la lampe, résulte d'une coupure, aplanie ensuite et adoucie par usure à sec avec de l'émeri fin. Telle est alors la sensibilité de l'appareil qu'elle met en évidence l'in'ime quantité de vapeur que la simple chaleur de la main dégage d'un cornet de papier qu'on lui présente. La vapeur émanant normalement de la main à la tempéra. ture ambiante par le fait des glandes sudoriparés se décèle à $1 \mathrm{~cm}$, de distance par une déviation du spot dont l'amplitude est d'ailleurs très différente suivant la région de la main présentée au tube. Alors que la face dorsale donne lieu à une déviation de 3 ou $4 \mathrm{~cm}$. (la règle étant à $108 \mathrm{~cm}$. du miroir), le bout d'un doigt donne lieu à un écart qui projette le spot hors de l'échelle.

Influence des effluves sur la résistance d'isolement des isolateurs. Note de M. F. NÈGRE, séance du 2 I avril r go8.

On sait que la résistance d'isolement d'un isolateur est définie par la relation $R I=V$, dans laquelle $V$ représente la tension appliquée à la gorge de l'isolateur, sa ferrure étant à la tension zéro, et l'intensité du courant qui va de la gorge de l'isolateur à la ferrure.

(*) On trouverd cette évaluation plus simple de $m$ en fonction de $N^{\prime}$, pour les valeurs de $N^{\prime}$ supérieures à 0,8 , c'est-à-dire pour les déversoits entièrement noyćs dont la nappe, de faible courbure, ne plonge plus sous le remous d'aval, mais est soulevée par lui, a la page I 8 du tome $L$ des Mémoires de l'Académie des Sciences, a la fin de ma Théorie approchée de l'écoulement de l'eau sur un déversoir en mince paroi et sans contraction latérale. 